\title{
Ethnobotanical study of medicinal plants from West Azerbaijan, Northwestern
} Iran

SAEIDEH ALIZADEH SALTEH ( $\nabla$ s.alizadeh@tabrizu.ac.ir)

University of Tabriz https://orcid.org/0000-0001-5849-7943

Mina Amani

University of Tabriz

\section{Research}

Keywords: Ethnopharmacology, West Azerbaijan, medicinal plants

Posted Date: August 24th, 2020

DOI: https://doi.org/10.21203/rs.3.rs-55496/v1

License: @ (i) This work is licensed under a Creative Commons Attribution 4.0 International License. Read Full License 


\section{Abstract \\ Background}

This study has identified the applications and local (Azari) and persian names of wild and cultivated plants collected for medical purposes by Azerian people of West Azerbaijan region in the Province of West Aerbaijan, Iran. The aim of this study is to collect information from local population concerning the use of medicinal plants of Khoy and determine the relative importance of the species including use value of species and the informant consensus factor (ICF) were surveyed and calculated in relation to medicinal plant use.

\section{Methods}

A field study had been carried out for a period of approximately 2 years (2014-2015). A questionnaire was administered to the local people, through face-toface interviews. Demographic characteristics of participants, names of the local plants, their utilized parts and preparation methods were asked. The plant species were collected as herbarium specimen. The collected data were used to calculate the ICF and the plant use values. 82 Plants were found to be used for medical purposes in study area.

\section{Results}

The results showed that the highest use value were recorded for the species Thymus kotschyanus Boiss. exhibited maximum use value (0.58), while the highest ICF was cited for Cold, flu, fever (0.61).

\section{Conclusions}

The results of this research showed that in the studied area the folk medicinal plants are still applied and evaluation of pharmacological activity for the indigenous medicinal plants is recommended.

\section{Introduction}

In spite of great progress in modern pharmacology and introduction of several new synthetic medicines, plants and their natural derivatives are widely used for various pharmacological purposes by the people in different regions1-6. These consumptions are due to the presence of different phytochemicals, which makes plants a major source of natural products for various medicinal applications. However, for more than 1000 years' people have used plants based on mouth to mouth information from past generations, without any detailed information regarding their phytochemical constituents (Hayta et al., 2014). Uncontrolled harvest of medicinal plants by local people has increased the risk of extinction of many species and subsequently the loss of local knowledge including instructions of those plants. Documentation of the indigenous knowledge through ethnobotanical studies is important for the conservation and utilization of biological resources. Therefore, establishing the local names and the indigenous uses of plants will be beneficial (Bagcl, 2000). In general, ethnobotany is the scientific investigations of plants that are used in indigenous culture for food, medicine, rituals, building, household implements, firewood, pesticides, clothing, shelter and other purposes (Ugulu, 2011). By revealing and recording the hidden folk medicinal uses of local plants, ethnobotany has become an important part of our world. Ethnobotany surveys include interviewing local people, using available data in the literature and the folklore of each region. Iran has admirable past regarding traditional medicines, especially medicinal Plant-based medicines (Naghibi et al., 2005). Historical evidence proves the fact that Iran is the most ancient civilization in using medicinal plants. Iran with 8000 plants species and 1727 endemic species, is one of the ten important sources of speciation in the world (Yousofi, 2007). Ethnobotany surveys were conducted in parts of the country (Ghorbani, 2005; Ghollassi Mood, 2008; Dolatkhahi et al., 2010; Iranmanesh et al., 2010; Sharififar et al., 2010). North west of Iran has a rich flora, due to its diverse climates and high number of ecological zones. This diversity in flora provides a rich source of medicinal plants, which has been utilized by Azerian people from the far past. The number of studies that have been undertaken to document and preserve medicinal plant knowledge in west Azerbaijan is few especially in Khoy region. For this purpose, we just found Study of West Azerbaijan medicinal plants that was conducted by Miraldi et al (2000). But they didn't evaluate the medicinal plants that grow in Khoy region and It's necessary to document biodiversity of medicinal plants that are used in folk medicinine in the study area. Khoy city possesses rich sources of different herbs. People have a ready access to them, especially in rural areas. Main objective of the current study therefore has been to compile and document information on the applications of plants by the people and the therapies offered by conventional healers in this area.

\section{Methodology}

Study area

Khoy is located north-west of Iran, West Azerbaijan province lying at $38^{\circ} 33^{\prime} 01^{\prime \prime} \mathrm{N} 44^{\circ} 57^{\prime} 08^{\prime \prime} \mathrm{E}$, with an average altitude $1139 \mathrm{~m}$ above sea level. It is nicknamed as the Sunflower city of Iran. In the past years, Khoy was the gateway of the Parthian Empire in the Northwest. Koppen-Geiger climate classification system classifies its climate as cold semi-arid. Qotur river which passes through the city, originates from high altitude of Turkish border. All around the region is surrounded by mountains like Iran's other western highlands. This area is along Mount Ararat (Agri Dagh). The city is located in the vicinity of mountains such as Chelekhaneh (north) Mount, Avrin Mount (southeast) and Aladagh mountain range in west of the city. It shares international border with Turkey (Van) at west. District Chaypare is located at north, Salmas at south and district Marand is allocated at west of Khoy. It is divided to four counties: Central District Municipality (Dizaj, Firuraq, Gharehsou, goharan Villages), Safayyeh District Municipality (Mmkhsh Khan, Aland, Sokman Abad district), Municipality of Ivughli Loading [MathJax]/jax/output/CommonHTML/jax.js ties Qotur (Zari and Qotur district) (Figure. 1). 
Interviews with local people

People Interviews were done during the busy hours of the known areas - visited by the citizens of West Azerbaijan and its villages. A questionnaire was administered to the local people, through face-to-face interviews. During the interviews, the demographic characteristics of the study participants, local names, utilized parts and preparation methods of the plants were recorded. The people who participated in the study were requested to indicate the wild plants they used.

\section{Plant Materials}

The field study was carried out over a period of approximately 2 years (2014-2015). During this period, information about the medicinal use of 72 wild and 20 cultivated plants were collected. The plants were pressed in the field and prepared for identification. Plants were identified using the standard text, Flora Iranica (Rechinger, 1965-2008) and Flora of Turkey and the East Aegean Islands (Davis, 1965-1985; Davis et al., 1988) and were compared with the specimens in Tabriz University Herbarium. The names of plant families were listed in alphabetic order.

\section{Data analysis}

The data was analyzed through different quantitative techniques. for this purpose, different approaches are considered for quantitative as well as qualitative analysis of ethnobotanical data. These approaches depend on the objectives of researcher, nature of the study and aim at objective evaluation of the reliability of the conclusions based on the data (Hoft et al., 1999). The indigenous medicinal information of plant species was analyzed using two different techniques: use value (UV) and informant consensus factor (ICF).

Informants consensus factor (ICF)

The importance of each species was represented by using different indices including, relative frequency of citation (FC), Informants consensus factor (ICF), and cultural importance index $(\mathrm{Cl})$. Frequency of citation $(\mathrm{FC})$ is defined as the number of informants who refer to a useful species. Informants consensus factor (ICF) index was used to determine the uniformity of the recorded information. At first the aliments were categorized and then all the citations were located into the related categories that each plant was claimed to affect. This index was calculated by using following formula:

$$
\mathrm{ICF}=\frac{\mathrm{N}_{\mathrm{ur}}-\mathrm{N}_{\mathrm{t}}}{\mathrm{N}_{\mathrm{ur}}-1}
$$

Where $\mathrm{N}_{\mathrm{ur}}$ and $\mathrm{N}_{\mathrm{t}}$ denote the number of use report in each use category and number of taxa taken as medicine, respectively. The higher the value of the ICF, the more informants agree on the use of the species in the use-category.

Use-value (UV)

The UV, a quantitative method to determine the relative importance of indigenous plant species was calculated using the following formula;

$$
\mathrm{UV}=\frac{\sum \mathrm{Ui}}{\mathrm{n}}
$$

Where, UV represents the use value of a species; Ui represents the number of uses mentioned by each informant for a given species; $\mathrm{n}$ is the total number of informants interviewed for a given species (Phillips and Gentry., 1993).

\section{Results And Discussion}

Demographic characteristics of study participants

Demographic characteristics of the respondents were determined and recorded through face to-face interviews. $55.8 \%$ of participants of surveyed people who had knowledge of plants were male and the rest were female. We interviewed 120 persons who are over the age of 25 . All females who use these plants are house- wives whereas $41.5 \%$ of males are farmers, and $29.2 \%$ of them are unemployed while others have various occupations. Demographical characteristics of interviewees according to the results, we obtained in the research, are presented in Table 1. 
Table 1

Demographic characteristics of the individuals

\begin{tabular}{|lll|}
\hline Demographical characteristics & Number & Percentage \\
\hline Age & & \\
\hline $25-34$ & 8 & 6.7 \\
\hline $35-49$ & 54 & 45.0 \\
\hline 50 above & 58 & 48.3 \\
\hline Sex & & \\
\hline Male & 67 & 55.8 \\
\hline Female & 53 & 44.2 \\
\hline Educational level & & \\
\hline Illiterate & 12 & 10.0 \\
\hline Primary school & 50 & 41.7 \\
\hline Secondary school & 25 & 20.8 \\
\hline High school & 19 & 15.8 \\
\hline University & 14 & 11.7 \\
\hline
\end{tabular}

A total of 82 plant taxa belonging to 31 families are reported which have been used for the cure of various ailments. An evaluation of the data from the Table 2: enlightens the fact that among the families Asteraceae (15 taxa), Lamiaceae ( 11 taxa) and Fabaceae ( 6 taxa) are the most dominant families, followed by Boraginaceae (6 taxa) (Fig. 2). Khoy ecologically is included in the Irano-Turanian phytogeographical region. Nearly 69 percent of plants distributed here are found in the flora of Iran. Ethnobotanical information of Sardasht people (southern part of West Azerbaijan province) has revealed that families mostly used here are approximately the same as recorded in our study presented here. The report by Azizi and Keshavarzi (2015) shows the most used plant families in that part are Asteraceae, Lamiaceae and Fabaceae each represented by 7, 6, 6 and 6 plant taxa. In a study from the Urmia city, West Azerbaijan province medicinal plants used for gastrointestinal ailments have been investigated. In all 138 plant species belonging to 52 families have been identified, dominant ones being Asteraceae (11 species) followed by Lamiaceae, and Rosaceae each with 5 and 3 species respectively (Bahmani et al., 2014). These families have also been reported in previous investigations undertaken in different regions of Iran such as; Kohghiluyeh va Boyer Ahmad (Mosaddegh et al., 2012), south of Kerman (Sadat et al., 2017) and Saravan region, Baluchistan (Sadegi et al., 2014). The reason why Asteraceae and Lamiaceae are mostly used in Iran and West Azerbaijan province is that they include hundreds of species with diverse medicinal application for different ailments. In addition to these, two other plant families as the most cited families in ethno-pharmacological studies in Iran during 2004-2016 are Apiaceae and Fabaceae; especially in the northern regions of Iran, Azerbaijan provinces (Sadegi et al., 2018). 
Table 2

Medicinal plants used in Khoy city for treatment of different ailments

\begin{tabular}{|c|c|c|c|c|c|c|c|c|}
\hline Nomber & Family & $\begin{array}{l}\text { Plant species } \\
\text { Voucher }\end{array}$ & $\begin{array}{l}\text { Vernacular } \\
\text { name }\end{array}$ & Persian name & $\begin{array}{l}\text { Plant } \\
\text { parts } \\
\text { used }\end{array}$ & Preparations & Medicinal uses & $\begin{array}{l}\text { Other } \\
\text { applications }\end{array}$ \\
\hline 1 & Alliaceae & $\begin{array}{l}\text { Allium cepa L.* } \\
\text { TUH-100 }\end{array}$ & Soğan & Piaz & Bulbs & $\begin{array}{l}\text { Infusion, } \\
\text { Raw }\end{array}$ & $\begin{array}{l}\text { fungal infection, } \\
\text { hypertension, } \\
\text { diabetes, cancer, } \\
\text { diuretic, rheumatism }\end{array}$ & Edible, food \\
\hline 2 & & $\begin{array}{l}\text { Allium sativum } \\
\text { L.* TUH-101 }\end{array}$ & Sarımsak & Sir & Bulbs & Powder, Raw & $\begin{array}{l}\text { Antihypertensive, } \\
\text { ringworm, } \\
\text { antiesthetic, diabetes, } \\
\text { cancer }\end{array}$ & $\begin{array}{l}\text { Edible, Pickle, } \\
\text { spice }\end{array}$ \\
\hline 3 & & $\begin{array}{l}\text { Allium } \\
\text { scorodoprasum } \\
\text { L. subsp. } \\
\text { rotundum (L.) } \\
\text { TUH-102 }\end{array}$ & Sirmuk & Sirmuk & $\begin{array}{l}\text { Bulbs, } \\
\text { Leaves }\end{array}$ & Infusion & $\begin{array}{l}\text { Antihypertensive, high } \\
\text { cholesterol }\end{array}$ & food \\
\hline 4 & Anacardiaceae & $\begin{array}{l}\text { Rhus coriaria L. } \\
\text { TUH-103 }\end{array}$ & Sumak & Sumak & Fruits & Infusion & $\begin{array}{l}\text { Antihypertensive, } \\
\text { diabetes, wound } \\
\text { healing }\end{array}$ & spice \\
\hline 5 & Apiaceae & $\begin{array}{l}\text { Falcaria } \\
\text { vulgaris Bernh. } \\
\text { TUH-104 }\end{array}$ & Gazayaghı & Ghazayaghı & $\begin{array}{l}\text { Aerial } \\
\text { parts }\end{array}$ & Decoction & $\begin{array}{l}\text { Stomach disease, } \\
\text { appetizer }\end{array}$ & $\begin{array}{l}\text { nourishment, } \\
\text { Additive in food }\end{array}$ \\
\hline 6 & & $\begin{array}{l}\text { Ferula } \\
\text { orientalis L. } \\
\text { TUH-105 }\end{array}$ & Chashir & - & $\begin{array}{l}\text { Aerial } \\
\text { parts }\end{array}$ & Decoction & $\begin{array}{l}\text { Hemorrhoids, } \\
\text { stomach disease }\end{array}$ & animal food \\
\hline 7 & & $\begin{array}{l}\text { Anethum } \\
\text { gravoelence* } \\
\text { TUH-106 }\end{array}$ & Shivid & $\begin{array}{l}\text { Shebet, } \\
\text { Shivid }\end{array}$ & $\begin{array}{l}\text { Aerial } \\
\text { parts }\end{array}$ & Decoction & $\begin{array}{l}\text { High cholesterol, } \\
\text { gastrointestinal } \\
\text { diseases, carminative }\end{array}$ & $\begin{array}{l}\text { Edible as a } \\
\text { vegetable }\end{array}$ \\
\hline 8 & & $\begin{array}{l}\text { Coriandrum } \\
\text { sativum* }^{\star} \\
\text { TUH-107 }\end{array}$ & Jashnish & Geshniz & Seeds & $\begin{array}{l}\text { Powder. } \\
\text { Infusion }\end{array}$ & $\begin{array}{l}\text { Stomached, diarrhea, } \\
\text { carminative, } \\
\text { increasing milk } \\
\text { production, appetizer }\end{array}$ & Edible, spice \\
\hline 9 & Asteraceae & $\begin{array}{l}\text { Achillea } \\
\text { tenuifolia. TUH- } \\
108\end{array}$ & Ingibir & Boomadaran & Leaves & Decoction & $\begin{array}{l}\text { Wound healing, } \\
\text { constipation }\end{array}$ & - \\
\hline 10 & & $\begin{array}{l}\text { Achillea } \\
\text { wilhelmsii. } \\
\text { TUH-109 }\end{array}$ & Boymadaran & Boomadaran & Flowers & $\begin{array}{l}\text { Decoction, } \\
\text { Infusion }\end{array}$ & $\begin{array}{l}\text { Blood coagulation, } \\
\text { constipation, } \\
\text { diabetes, } \\
\text { hemorrhoids, } \\
\text { hypertension, }\end{array}$ & - \\
\hline 11 & & $\begin{array}{l}\text { Arctium minus } \\
\text { (Hill.) Bernh } \\
\text { TUH-110 }\end{array}$ & $\begin{array}{l}\text { Pitrakh, } \\
\text { Gangal }\end{array}$ & Baba adam & $\begin{array}{l}\text { Aerial } \\
\text { parts, } \\
\text { Leaves }\end{array}$ & Decoction & $\begin{array}{l}\text { Cold and flu, } \\
\text { hemorrhoids, } \\
\text { rheumatism, wound } \\
\text { healing }\end{array}$ & - \\
\hline 12 & Asteraceae & $\begin{array}{l}\text { Artemisia } \\
\text { absinthium L. } \\
\text { TUH-111 }\end{array}$ & Yoshan & Afsantin & $\begin{array}{l}\text { Aerial } \\
\text { parts }\end{array}$ & $\begin{array}{l}\text { Infusion, } \\
\text { Raw }\end{array}$ & $\begin{array}{l}\text { Coughing, headache, } \\
\text { stomachache, wound } \\
\text { healing }\end{array}$ & - \\
\hline 13 & & $\begin{array}{l}\text { Echinops } \\
\text { pungens. TUH- } \\
112\end{array}$ & Tippuz & $\begin{array}{l}\text { Shekartighal, } \\
\text { Ghgandrunak }\end{array}$ & $\begin{array}{l}\text { Aerial } \\
\text { parts }\end{array}$ & Decoction & $\begin{array}{l}\text { Respiratory diseases, } \\
\text { expectorant, cancer }\end{array}$ & - \\
\hline 14 & & $\begin{array}{l}\text { Gundelia } \\
\text { tournefortii. } \\
\text { TUH-113 }\end{array}$ & Kenger & Kangar & $\begin{array}{l}\text { Roots, } \\
\text { Latex }\end{array}$ & Infusion & Diabetes, diarrhea & Food \\
\hline 15 & & $\begin{array}{l}\text { Helianthus } \\
\text { annuus L. * } \\
\text { TUH-114 }\end{array}$ & Shalgam & $\begin{array}{l}\text { Sibzamini } \\
\text { torshi }\end{array}$ & $\begin{array}{l}\text { Fruits, } \\
\text { Leaves, } \\
\text { Seeds }\end{array}$ & Raw & $\begin{array}{l}\text { Respiratory tract } \\
\text { diseases and flu, } \\
\text { bronchitis }\end{array}$ & Edible, Pickle \\
\hline 16 & & $\begin{array}{l}\text { Helichrysum } \\
\text { arenarium (L.) } \\
\text { Moench. TUH- } \\
115\end{array}$ & Sari gul & Gole bimarg & $\begin{array}{l}\text { Aerial } \\
\text { parts }\end{array}$ & Infusion & $\begin{array}{l}\text { Colds and flu, } \\
\text { gastrointestinal } \\
\text { diseases, diabetes, } \\
\text { hypercholesterole }\end{array}$ & - \\
\hline 17 & & $\begin{array}{l}\text { Matricaria } \\
\text { chamomilla L. } \\
\text { TUH-116 }\end{array}$ & Babanah & Babooneh & $\begin{array}{l}\text { Aerial } \\
\text { parts }\end{array}$ & Infusion & $\begin{array}{l}\text { Gastrointestinal } \\
\text { diseases, headache, } \\
\text { respiratory tract } \\
\text { diseases, flu, cancer, } \\
\text { antiinflammation }\end{array}$ & - \\
\hline
\end{tabular}




\begin{tabular}{|c|c|c|c|c|c|c|c|c|}
\hline Nomber & Family & $\begin{array}{l}\text { Plant species } \\
\text { Voucher }\end{array}$ & $\begin{array}{l}\text { Vernacular } \\
\text { name }\end{array}$ & Persian name & $\begin{array}{l}\text { Plant } \\
\text { parts } \\
\text { used }\end{array}$ & Preparations & Medicinal uses & $\begin{array}{l}\text { Other } \\
\text { applications }\end{array}$ \\
\hline 18 & & $\begin{array}{l}\text { Onopordum } \\
\text { acanthium L. } \\
\text { TUH-117 }\end{array}$ & Gush gonmaz & $\begin{array}{l}\text { Kare } \\
\text { zanbaba }\end{array}$ & Flowers & Decoction & Hemorrhoids & - \\
\hline 19 & & $\begin{array}{l}\text { Senecio } \\
\text { vulgaris L. } \\
\text { TUH-118 }\end{array}$ & Kanarya out & $\begin{array}{l}\text { Pirgiah, zolfe } \\
\text { pir }\end{array}$ & $\begin{array}{l}\text { Flowers, } \\
\text { Seeds }\end{array}$ & Infusion & Bronchitis, diuretic & - \\
\hline 20 & & $\begin{array}{l}\text { Silybum } \\
\text { marianum (L.) } \\
\text { Gaertn. TUH- } \\
119\end{array}$ & Maryam tikani & $\begin{array}{l}\text { Kharmaryam, } \\
\text { Maritighal }\end{array}$ & $\begin{array}{l}\text { Stylus, } \\
\text { Seeds }\end{array}$ & $\begin{array}{l}\text { Raw, } \\
\text { Infusion, } \\
\text { Decoction }\end{array}$ & $\begin{array}{l}\text { Rheumatism, } \\
\text { appetizer, diuretic }\end{array}$ & - \\
\hline 21 & & $\begin{array}{l}\text { Trogopogon } \\
\text { graminifolius. } \\
\text { TUH-120 }\end{array}$ & Yelmih & Sheng & $\begin{array}{l}\text { Aerial } \\
\text { parts }\end{array}$ & $\begin{array}{l}\text { Decoction, } \\
\text { Raw }\end{array}$ & $\begin{array}{l}\text { Gastrointestinal } \\
\text { diseases, } \\
\text { rheumatism, } \\
\text { appetizer }\end{array}$ & - \\
\hline 22 & & $\begin{array}{l}\text { Xanthium } \\
\text { spinosum L. } \\
\text { TUH-121 }\end{array}$ & Aji tikan & Togh, mastak & Seeds & Decoction & $\begin{array}{l}\text { Kidney pain, } \\
\text { gastrointestinal } \\
\text { diseases }\end{array}$ & - \\
\hline 23 & & $\begin{array}{l}\text { Xanthium } \\
\text { strumarium L. } \\
\text { TUH-122 }\end{array}$ & pitrakh & Zardan & Fruits & Decoction & Hemorrhoids, asthma & - \\
\hline 24 & Boraginaceae & $\begin{array}{l}\text { Anchusa } \\
\text { azurea Mill. var. } \\
\text { azurea. TUH- } \\
123\end{array}$ & Gowaban & Gavzaban & Leaves & Infusion & $\begin{array}{l}\text { Sedative, flu, } \\
\text { stomach, wound } \\
\text { healing, } \\
\text { antiinflammatory }\end{array}$ & \\
\hline 25 & Boraginaceae & $\begin{array}{l}\text { Capsella bursa- } \\
\text { pastoris (L.) } \\
\text { TUH-124 }\end{array}$ & Gush appayi & Kise keshish & $\begin{array}{l}\text { Aerial } \\
\text { parts }\end{array}$ & Raw & Impotence, appetizer & Edible \\
\hline 26 & & $\begin{array}{l}\text { Descurainia } \\
\text { sophia L. TUH- } \\
125\end{array}$ & Shovaran & Khakshir & Seeds & $\begin{array}{l}\text { Syrup, } \\
\text { Decoction }\end{array}$ & $\begin{array}{l}\text { Diarrhea and } \\
\text { abdominal pain, sore } \\
\text { throat }\end{array}$ & $\begin{array}{l}\text { Edible, Air } \\
\text { Disinfectants }\end{array}$ \\
\hline 27 & & $\begin{array}{l}\text { Lepidium draba } \\
\text { L. TUH-126 }\end{array}$ & Dit otu & Ozmak & $\begin{array}{l}\text { Aerial } \\
\text { parts }\end{array}$ & Decoction & Cold and fever & - \\
\hline 28 & & $\begin{array}{l}\text { Nasturtium } \\
\text { officinale W. T. } \\
\text { Aiton. TUH-127 }\end{array}$ & Bulagh otu & $\begin{array}{l}\text { Alaf } \\
\text { cheshmeh, } \\
\text { taretizak }\end{array}$ & $\begin{array}{l}\text { Aerial } \\
\text { parts }\end{array}$ & Infusion & $\begin{array}{l}\text { Gastrointestinal } \\
\text { diseases, diuretic, } \\
\text { diabetes disease, } \\
\text { digestive, appetizer }\end{array}$ & - \\
\hline 29 & & $\begin{array}{l}\text { Raphanus } \\
\text { raphanistrum } \\
\text { L.* TUH-128 }\end{array}$ & turp & Torob & $\begin{array}{l}\text { Leaves, } \\
\text { Roots }\end{array}$ & Raw & $\begin{array}{l}\text { Appetizer, liver } \\
\text { protecting, muscular } \\
\text { pains }\end{array}$ & - \\
\hline 30 & Brassicaceae & $\begin{array}{l}\text { Sinapis } \\
\text { arvensis L. } \\
\text { TUH-129 }\end{array}$ & Khardal, turpak & Khardal & Seeds & Decoction & $\begin{array}{l}\text { Bronchitis, } \\
\text { pneumonitis }\end{array}$ & $\begin{array}{l}\text { Spice, } \\
\text { Disinfectants }\end{array}$ \\
\hline 31 & & $\begin{array}{l}\text { Thlaspi } \\
\text { perfoliatum L. } \\
\text { TUH-130 }\end{array}$ & Goddume & Ghoddume & $\begin{array}{l}\text { Aerial } \\
\text { parts }\end{array}$ & $\begin{array}{l}\text { Infusion, } \\
\text { Raw }\end{array}$ & Painkiller & \\
\hline 32 & Capparidaceae & $\begin{array}{l}\text { Capparis } \\
\text { spinosa L. } \\
\text { TUH-131 }\end{array}$ & $\begin{array}{l}\text { Dagh } \\
\text { garpouzu }\end{array}$ & Kabar, Lagaji & $\begin{array}{l}\text { Fruits, } \\
\text { Flowers }\end{array}$ & $\begin{array}{l}\text { Raw, } \\
\text { Decoction }\end{array}$ & $\begin{array}{l}\text { Joint pain such as } \\
\text { back pain and leg }\end{array}$ & Edible, Pickle \\
\hline 33 & Caryophyllaceae & $\begin{array}{l}\text { Stellaria media } \\
\text { (L.) Vill. TUH- } \\
132\end{array}$ & Jinjilim & $\begin{array}{l}\text { Gandomak, } \\
\text { Daneye } \\
\text { ghanari }\end{array}$ & $\begin{array}{l}\text { Aerial } \\
\text { parts }\end{array}$ & $\begin{array}{l}\text { Infusion, } \\
\text { Raw }\end{array}$ & Hypercholesterolemia & - \\
\hline 34 & chenopodiaceae & $\begin{array}{l}\text { Beta vulgaris } \\
\text { L.* TUH-133 }\end{array}$ & Bine & Choghondar & Leaves & $\begin{array}{l}\text { Infusion, } \\
\text { Raw }\end{array}$ & Wound healing & $\begin{array}{l}\text { Sugar } \\
\text { production }\end{array}$ \\
\hline 35 & & $\begin{array}{l}\text { Chenopodium } \\
\text { album. TUH- } \\
134\end{array}$ & Yaghliga & Salmak & $\begin{array}{l}\text { Aerial } \\
\text { parts }\end{array}$ & Decoction & $\begin{array}{l}\text { Helminth, } \\
\text { antiinflammation, } \\
\text { toothache }\end{array}$ & food \\
\hline 36 & Cucurbitaceae & $\begin{array}{l}\text { Cucurbita } \\
\text { moschata *. } \\
\text { TUH-135 }\end{array}$ & Bal kabağı & Kadoo & Fruits & $\begin{array}{l}\text { Raw, } \\
\text { Decoction, } \\
\text { Infusion }\end{array}$ & Diuretic, carminative & - \\
\hline 37 & & $\begin{array}{l}\text { Cucurbita } \\
\text { pepo*. TUH- } \\
136\end{array}$ & Kado & $\begin{array}{l}\text { Kadoo } \\
\text { pooste } \\
\text { kaghazi }\end{array}$ & Seeds & Oil, Raw & $\begin{array}{l}\text { Hyperplasia, skin } \\
\text { diseases, wound } \\
\text { healing }\end{array}$ & - \\
\hline
\end{tabular}




\begin{tabular}{|c|c|c|c|c|c|c|c|c|}
\hline Nomber & Family & $\begin{array}{l}\text { Plant species } \\
\text { Voucher }\end{array}$ & $\begin{array}{l}\text { Vernacular } \\
\text { name }\end{array}$ & Persian name & $\begin{array}{l}\text { Plant } \\
\text { parts } \\
\text { used }\end{array}$ & Preparations & Medicinal uses & $\begin{array}{l}\text { Other } \\
\text { applications }\end{array}$ \\
\hline 38 & Elaegnaceae & $\begin{array}{l}\text { Elaeagnus } \\
\text { angustifolia } \\
\text { L.*. TUH-137 }\end{array}$ & Ide & Senjed & Fruits & Raw, Powder & $\begin{array}{l}\text { Osteoporosis, } \\
\text { stomachache }\end{array}$ & $\begin{array}{l}\text { Edible/livestock } \\
\text { forage }\end{array}$ \\
\hline 39 & Euphorbiaceae & $\begin{array}{l}\text { Euphorbia } \\
\text { macroclada } \\
\text { Boiss. TUH-138 }\end{array}$ & Sütlyan & $\begin{array}{l}\text { Farfion, } \\
\text { shirsag }\end{array}$ & Latex & Infusion & Curing warts & - \\
\hline 40 & & $\begin{array}{l}\text { Ricinus } \\
\text { communis L.* } \\
\text { TUH-139 }\end{array}$ & Gharchah & Karchak & Leaves & Poultice & Rheumatism & - \\
\hline 41 & Fabaceae & $\begin{array}{l}\text { Alhagi } \\
\text { pseudalhagi } \\
\text { (M. Bieb.). TUH- } \\
140\end{array}$ & Dava tikani & Kharshotor & $\begin{array}{l}\text { Aerial } \\
\text { parts }\end{array}$ & Decoction & Kidney stones & - \\
\hline 42 & & $\begin{array}{l}\text { Astragalus } \\
\text { gummifer. } \\
\text { TUH-141 }\end{array}$ & Gavan & Gavan & $\begin{array}{l}\text { Leaves, } \\
\text { Latex }\end{array}$ & Decoction & $\begin{array}{l}\text { Heart diseases, } \\
\text { cough, hemorrhoids, } \\
\text { diabetes disease }\end{array}$ & $\begin{array}{l}\text { Hair } \\
\text { Amplifier } \\
\text { (Katira) }\end{array}$ \\
\hline 43 & & $\begin{array}{l}\text { Glycyrrhiza } \\
\text { glabra L. TUH- } \\
142\end{array}$ & Shirin bayan & Shirin bayan & $\begin{array}{l}\text { Roots, } \\
\text { Aerial } \\
\text { parts }\end{array}$ & $\begin{array}{l}\text { Decoction, } \\
\text { Infusion }\end{array}$ & $\begin{array}{l}\text { Treat colds, stomach } \\
\text { pain, bone fractures, } \\
\text { sedative, stomach } \\
\text { and kidney disorders }\end{array}$ & - \\
\hline 44 & & $\begin{array}{l}\text { Medicago } \\
\text { sativa L.* }{ }^{\text {TUH- }} \\
143\end{array}$ & Yonja & Yonje & Leaves & $\begin{array}{l}\text { Infusion, } \\
\text { Decoction }\end{array}$ & $\begin{array}{l}\text { Strengthening } \\
\text { children, weight gain }\end{array}$ & Edible \\
\hline 45 & & $\begin{array}{l}\text { Melilotus } \\
\text { officinalis. } \\
\text { TUH-144 }\end{array}$ & Sari yonja & Yonje-ye zard & $\begin{array}{l}\text { Aerial } \\
\text { parts }\end{array}$ & $\begin{array}{l}\text { Infusion, } \\
\text { Powder }\end{array}$ & $\begin{array}{l}\text { Sedative, kidney } \\
\text { stones, astringent, } \\
\text { diuretic, burns healing }\end{array}$ & - \\
\hline 46 & & $\begin{array}{l}\text { Trifolium } \\
\text { pratense L. } \\
\text { TUH-145 }\end{array}$ & $\begin{array}{l}\text { Ush gulakh, } \\
\text { Dolbarjin }\end{array}$ & Shabdar & $\begin{array}{l}\text { Aerial } \\
\text { parts }\end{array}$ & Decoction & Diarrhea, stomach & $\begin{array}{l}\text { Livestock, } \\
\text { forage }\end{array}$ \\
\hline 47 & Fumariaceae & $\begin{array}{l}\text { Fumaria } \\
\text { parviflora Lam. } \\
\text { TUH-146 }\end{array}$ & Shatereh & Shatereh & $\begin{array}{l}\text { Fruits, } \\
\text { Aerial } \\
\text { parts }\end{array}$ & $\begin{array}{l}\text { Infusion, } \\
\text { Decoction }\end{array}$ & Abdominal pain & Edible \\
\hline 48 & Iridaceae & $\begin{array}{l}\text { Iris germanica } \\
\text { L. TUH-147 }\end{array}$ & Nevruz çiçeği & Zanbagh & Roots & Crushed & Toothache & \\
\hline 49 & Juglandaceae & $\begin{array}{l}\text { Juglans regia } \\
\text { L.* TUH-148 }\end{array}$ & Javiz & Gerdoo & $\begin{array}{l}\text { Seeds, } \\
\text { Aearial } \\
\text { parts }\end{array}$ & $\begin{array}{l}\text { Decoction, } \\
\text { Powder }\end{array}$ & $\begin{array}{l}\text { Diabetes and blanch } \\
\text { teeth }\end{array}$ & $\begin{array}{l}\text { Edible, hair } \\
\text { color and } \\
\text { dyeing yarn }\end{array}$ \\
\hline 50 & Lamiaceae & $\begin{array}{l}\text { Eremostachys } \\
\text { laciniata. TUH- } \\
149\end{array}$ & Chelle daghi & Chelle daghi & $\begin{array}{l}\text { Aerial } \\
\text { parts, } \\
\text { Leaves, } \\
\text { Roots }\end{array}$ & $\begin{array}{l}\text { Poultice, } \\
\text { Powder }\end{array}$ & $\begin{array}{l}\text { Rheumatism, joint } \\
\text { pains, wound healing }\end{array}$ & \\
\hline 51 & & $\begin{array}{l}\text { Leonorus } \\
\text { cardica L. TUH- } \\
150\end{array}$ & ot & Dime shir & $\begin{array}{l}\text { Aerial } \\
\text { parts }\end{array}$ & Decoction & $\begin{array}{l}\text { Amenorrhea, } \\
\text { digestive, cardiac } \\
\text { diseases }\end{array}$ & \\
\hline 52 & & $\begin{array}{l}\text { Mentha } \\
\text { longifolia (L.) } \\
\text { Huds. TUH-151 }\end{array}$ & Yarpuz & Pooneh & Leaves & $\begin{array}{l}\text { Decoction, } \\
\text { Powder }\end{array}$ & $\begin{array}{l}\text { Cold and flu, } \\
\text { antispasmodic, } \\
\text { constipation, cold } \\
\text { and fever, } \\
\text { gastrointestinal } \\
\text { diseases }\end{array}$ & Edible \\
\hline 53 & & $\begin{array}{l}\text { Mentha spicata } \\
\text { L. subsp. } \\
\text { Spicata*. TUH- } \\
152\end{array}$ & Nane & Naana & $\begin{array}{l}\text { Aerial } \\
\text { parts }\end{array}$ & $\begin{array}{l}\text { Infusion, } \\
\text { Decoction }\end{array}$ & $\begin{array}{l}\text { Cold and flu, vomit, } \\
\text { digestive, Diarrhea, } \\
\text { Gastrointestinal } \\
\text { diseases }\end{array}$ & Edible, Flavors \\
\hline 54 & & $\begin{array}{l}\text { Ocimum } \\
\text { basilicum L.* } \\
\text { TUH-153 }\end{array}$ & Reyhan & Reyhan & $\begin{array}{l}\text { Aerial } \\
\text { parts }\end{array}$ & $\begin{array}{l}\text { Infusion, } \\
\text { Decoction }\end{array}$ & Stomach, appetizer & $\begin{array}{l}\text { Edible as a } \\
\text { vegetable }\end{array}$ \\
\hline 55 & & $\begin{array}{l}\text { Salvia } \\
\text { tomentosa Mill. } \\
\text { TUH-154 }\end{array}$ & Maryam gulu & Maryam goli & Leaves & $\begin{array}{l}\text { Infusion, } \\
\text { Decoction }\end{array}$ & $\begin{array}{l}\text { Flu, bronchitis, } \\
\text { diarrhea, asthma, } \\
\text { rheumatism, } \\
\text { toothache, } \\
\text { gastrointestinal } \\
\text { diseases. }\end{array}$ & - \\
\hline
\end{tabular}




\begin{tabular}{|c|c|c|c|c|c|c|c|c|}
\hline Nomber & Family & $\begin{array}{l}\text { Plant species } \\
\text { Voucher }\end{array}$ & $\begin{array}{l}\text { Vernacular } \\
\text { name }\end{array}$ & Persian name & $\begin{array}{l}\text { Plant } \\
\text { parts } \\
\text { used }\end{array}$ & Preparations & Medicinal uses & $\begin{array}{l}\text { Other } \\
\text { applications }\end{array}$ \\
\hline 56 & & $\begin{array}{l}\text { Satureja } \\
\text { hortensis L.* } \\
\text { TUH-155 }\end{array}$ & Marza & Marze & $\begin{array}{l}\text { Aerial } \\
\text { parts }\end{array}$ & Infusion & $\begin{array}{l}\text { Antihypertensive, } \\
\text { cardiac disorder, } \\
\text { gastric and digestive } \\
\text { disorders }\end{array}$ & $\begin{array}{l}\text { Edible as a } \\
\text { vegetable, food }\end{array}$ \\
\hline 57 & & $\begin{array}{l}\text { Stachys inflate. } \\
\text { TUH-156 }\end{array}$ & $\begin{array}{l}\text { Poolaki, } \\
\text { Maryam } \\
\text { nokhodu }\end{array}$ & Poolaki & $\begin{array}{l}\text { Aerial } \\
\text { parts }\end{array}$ & Infusion & Cold, hypertension & - \\
\hline 58 & & $\begin{array}{l}\text { Stachys } \\
\text { lavandulifolia } \\
\text { Vahl. TUH-157 }\end{array}$ & Tuhliga & Chaye koohi & $\begin{array}{l}\text { Aerial } \\
\text { parts, }\end{array}$ & Infusion & Cold, infection & - \\
\hline 59 & & $\begin{array}{l}\text { Teucrium } \\
\text { polium L. TUH- } \\
158\end{array}$ & Kalpooreh & Kalpooreh & Flowers & $\begin{array}{l}\text { Infusion, } \\
\text { Smoke }\end{array}$ & $\begin{array}{l}\text { Ear infection, diarrhea } \\
\text { in infants, Vaginal } \\
\text { Infection }\end{array}$ & - \\
\hline 60 & & $\begin{array}{l}\text { Thymus } \\
\text { kotschyanus } \\
\text { Boiss. TUH-159 }\end{array}$ & Kahlik otu & Avishan & $\begin{array}{l}\text { Aerial } \\
\text { parts, } \\
\text { Leaves }\end{array}$ & Decoction & $\begin{array}{l}\text { Cold, gastritis, } \\
\text { antiinflammatory, } \\
\text { cancer,, diabetes, } \\
\text { hypertension }\end{array}$ & - \\
\hline 61 & Malvaceae & $\begin{array}{l}\text { Alcea kurdica } \\
\text { (Schlecht) Alef. } \\
\text { TUH-160 }\end{array}$ & Khatmi & Khatmi & $\begin{array}{l}\text { Flo, } \\
\text { Roots }\end{array}$ & Decoction & $\begin{array}{l}\text { Pneumonia, cold and } \\
\text { flu }\end{array}$ & - \\
\hline 62 & & $\begin{array}{l}\text { Ficus carica L.* } \\
\text { TUH-161 }\end{array}$ & Anjir & Anjir & $\begin{array}{l}\text { Fruits, } \\
\text { Latex, }\end{array}$ & Raw & $\begin{array}{l}\text { Constipation, laxative, } \\
\text { warts }\end{array}$ & - \\
\hline 63 & & $\begin{array}{l}\text { Malva neglecta } \\
\text { L. TUH-162 }\end{array}$ & Aman komanji & Panirak & $\begin{array}{l}\text { Flowers, } \\
\text { Leaves }\end{array}$ & Infusion & $\begin{array}{l}\text { Gastrointestinal } \\
\text { diseases, bronchitis, } \\
\text { diabetes, } \\
\text { hypercholesterolemia. }\end{array}$ & - \\
\hline 64 & Papaveraceae & $\begin{array}{l}\text { Papaver rhoeas } \\
\text { L. TUH-163 }\end{array}$ & Shaghayegh & Shaghayegh & $\begin{array}{l}\text { Aerial } \\
\text { parts }\end{array}$ & Decoction & Antitussive, sedative & - \\
\hline 65 & Plantaginaceae & $\begin{array}{l}\text { Plantago } \\
\text { lanceolata L. } \\
\text { TUH-164 }\end{array}$ & bagh yarpağı & Barhang & Leaves & Infusion & $\begin{array}{l}\text { Antipyretic, wound } \\
\text { healing }\end{array}$ & - \\
\hline 66 & & $\begin{array}{l}\text { Plantago major } \\
\text { L. TUH-165 }\end{array}$ & Bizowsha & Barhang & Seeds & $\begin{array}{l}\text { Syrup, } \\
\text { Decoction }\end{array}$ & $\begin{array}{l}\text { Abdominal pains, } \\
\text { hemorrhoids, } \\
\text { constipation, }\end{array}$ & - \\
\hline 67 & Poaceae & $\begin{array}{l}\text { Hordeum } \\
\text { vulgare L.* } \\
\text { TUH-166 }\end{array}$ & Arpa & Jo & $\begin{array}{l}\text { Aerial } \\
\text { parts, } \\
\text { Seeds }\end{array}$ & $\begin{array}{l}\text { Infusion, } \\
\text { Powder }\end{array}$ & $\begin{array}{l}\text { Intestinal regulatory, } \\
\text { diarrhea, appetizer }\end{array}$ & - \\
\hline 68 & & $\begin{array}{l}\text { Zea mays L.* } \\
\text { TUH-167 }\end{array}$ & Maka & Zorrat & $\begin{array}{l}\text { Fruits, } \\
\text { Stylus }\end{array}$ & Decoction & $\begin{array}{l}\text { Infection of urinary } \\
\text { system, heart tonic, } \\
\text { kidney stones }\end{array}$ & - \\
\hline 69 & Polygonaceae & $\begin{array}{l}\text { Rheum ribes } L \text {. } \\
\text { TUH-168 }\end{array}$ & Ushgun & Rivas & $\begin{array}{l}\text { Aerial } \\
\text { parts, } \\
\text { Leaves }\end{array}$ & Infusion & Diabetes disease & - \\
\hline 70 & & $\begin{array}{l}\text { Rumex crispus } \\
\text { L. TUH- } 169\end{array}$ & Avalih & Torshak & Leaves & Infusion & Diabetes disease & - \\
\hline 71 & Portulaceae & $\begin{array}{l}\text { Portulaca } \\
\text { oleracea L. } \\
\text { TUH-170 }\end{array}$ & Parpine & Khorfe & $\begin{array}{l}\text { Aerial } \\
\text { parts }\end{array}$ & Infusion & Diabetes, diuretic & - \\
\hline 72 & Ranunculaceae & $\begin{array}{l}\text { Consolida } \\
\text { orientalis. TUH- } \\
171\end{array}$ & $\begin{array}{l}\text { Akin out, } \\
\text { Bughda otu }\end{array}$ & $\begin{array}{l}\text { Zaban dar } \\
\text { ghafa }\end{array}$ & Seeds & Crushed & Wound healing & - \\
\hline 73 & Rosaceae & $\begin{array}{l}\text { Rosa canina L. } \\
\text { TUH-172 }\end{array}$ & İtburnu & Nastaran & $\begin{array}{l}\text { Flowers, } \\
\text { Fruits }\end{array}$ & Infusion & $\begin{array}{l}\text { Antitussive, cold, } \\
\text { diabetes disease }\end{array}$ & - \\
\hline 74 & & $\begin{array}{l}\text { Cydonia } \\
\text { oblonga Mill.* } \\
\text { TUH-173 }\end{array}$ & Heyva & Beh & $\begin{array}{l}\text { Seeds, } \\
\text { Fruits, } \\
\text { Leaves }\end{array}$ & $\begin{array}{l}\text { Raw, } \\
\text { Decoction, } \\
\text { Infusion }\end{array}$ & $\begin{array}{l}\text { Cold and flu, } \\
\text { bronchitis, asthma, } \\
\text { diuretic, } \\
\text { gastrointestinal } \\
\text { diseases. }\end{array}$ & - \\
\hline 75 & & $\begin{array}{l}\text { Rosa } \\
\text { damascena* } \\
\text { TUH-174 }\end{array}$ & Ghizil gul & $\begin{array}{l}\text { Gole } \\
\text { mohammadi }\end{array}$ & Flowers & $\begin{array}{l}\text { Oil, Raw, } \\
\text { Infusion }\end{array}$ & $\begin{array}{l}\text { Burns and wounds } \\
\text { healing, sedative, } \\
\text { stomach } \\
\text { and reflux, Laxative }\end{array}$ & $\begin{array}{l}\text { Perfume in } \\
\text { Funerals }\end{array}$ \\
\hline
\end{tabular}




\begin{tabular}{|c|c|c|c|c|c|c|c|c|}
\hline Nomber & Family & $\begin{array}{l}\text { Plant species } \\
\text { Voucher }\end{array}$ & $\begin{array}{l}\text { Vernacular } \\
\text { name }\end{array}$ & Persian name & $\begin{array}{l}\text { Plant } \\
\text { parts } \\
\text { used }\end{array}$ & Preparations & Medicinal uses & $\begin{array}{l}\text { Other } \\
\text { applications }\end{array}$ \\
\hline 76 & Salicaceae & $\begin{array}{l}\text { Salix alba L. } \\
\text { TUH-175 }\end{array}$ & Suwidi & Bid & $\begin{array}{l}\text { Aerial } \\
\text { parts, } \\
\text { Leaves }\end{array}$ & Decoction & $\begin{array}{l}\text { Vaginal itching, } \\
\text { athlete's foot }\end{array}$ & Landscape \\
\hline 77 & Scrophulariaceae & $\begin{array}{l}\text { Verbascum } \\
\text { spp. TUH-176 }\end{array}$ & Sıghırkuyrughu & $\begin{array}{l}\text { Khargooshak, } \\
\text { Gole mahoor }\end{array}$ & $\begin{array}{l}\text { Aerial } \\
\text { parts }\end{array}$ & Infusion & $\begin{array}{l}\text { Hemorrhoids, urinary } \\
\text { tract infection }\end{array}$ & - \\
\hline 78 & Solanaceae & $\begin{array}{l}\text { Datura } \\
\text { stramonium L. } \\
\text { TUH-177 }\end{array}$ & $\begin{array}{l}\text { Tatoore, bat } \\
\text { bat }\end{array}$ & Tatoore & $\begin{array}{l}\text { Aerial } \\
\text { parts, } \\
\text { Seeds }\end{array}$ & Infusion & $\begin{array}{l}\text { Eye problems, } \\
\text { asthma, narcotic, } \\
\text { nerve sedative, } \\
\text { rheumatism pain }\end{array}$ & - \\
\hline 79 & & $\begin{array}{l}\text { Hyoscyamus } \\
\text { niger L. TUH- } \\
178\end{array}$ & Pat pat & $\begin{array}{l}\text { Bangdaneh, } \\
\text { bazrolbanj }\end{array}$ & Seeds & Poultice & $\begin{array}{l}\text { Ear diseases, } \\
\text { sinusitis, addiction } \\
\text { disuse }\end{array}$ & - \\
\hline 80 & Urticaceae & $\begin{array}{l}\text { Urtica dioica L. } \\
\text { TUH-179 }\end{array}$ & Dalama & Gazaneh & Leaves & $\begin{array}{l}\text { Decoction, } \\
\text { Raw }\end{array}$ & $\begin{array}{l}\text { Cardiovascular } \\
\text { disease, cough, } \\
\text { diabetes, MS }\end{array}$ & Food \\
\hline 81 & Zygophyllaceae & $\begin{array}{l}\text { Peganum } \\
\text { harmala L. } \\
\text { TUH-180 }\end{array}$ & Uzarrih & Esphand & Fruits & Raw, Smoke & Fever, disinfectants & Air freshener \\
\hline 82 & & $\begin{array}{l}\text { Tribulus } \\
\text { terrestris L. } \\
\text { TUH-181 }\end{array}$ & Damirtikani & Kharkhasak & Fruits & Decoction & Kidney stones & - \\
\hline
\end{tabular}

Plant parts used

Different medicinal plant parts used for curing diverse aliments included aerial parts, seed, leaves, tuber, root, stem, gum, flower, fruit, and the whole plant. Most frequently used parts are aerial parts (32\%), followed by leaves (20\%), Seeds (13\%), and Fruits (12\%) (Figure. 3 ). The preferred parts of some medicinal plants may have high concentration of bioactive compounds, leading to efficient responses in curing the concerned aliment. It has been documented that families like, Asteraceae, Lamiaceae and Fabaceae usually as such these parts are preferred by local people in different regions of Iran11, 14, 23. Second reason why aerial parts and leaves are preferred is that they are easier to harvest as compared to underground parts. Moreover, aerial parts are accessible in most of year than flowers, seeds or fruits, which are accessible only in the reproductive stages of plants, limiting harvesting time to a short period. Another reason is associated with harvesting time of the plants, since in Khoy region people mainly harvest the medicinal plants in late spring, when herbs are in their vegetative stage so that there would be no flower or seed to be harvested.

Preparation methods and mode of application

Most of the native people make medicines by using simple preparation of plant parts. The most common preparation method which is reported by indigenous people in Khoy were decoction, infusion and powder which are prepared by the patients themselves. Water is the dominated supporting agent with herbal therapy. Infusions were the most used method in preparation of plants. It is applied by soaking plant part in hot water for the time specified as a day. Decoctions were the second common method that includes boiling a specific part of plant in water until it reaches its half the initial volume. Among preparation methods, those which lead to an orally consumable products are preferred (Mood, 2008; Brandao et al., 2012; Sadeghi et al., 2014). It was observed that native people of this region used endogenous plants after drying. This method is preferred because they can store these dried medicinal plants in a house room and use it for all year round, when they need. In West Azerbaijan, the custom of preparing distillates at home is a widespread tradition named "Araghgiri" that produce aromatic water with homemade instruments named "Neygazan". For example, distillate obtained from Mentha, used to treat abdominal pains and also used as carminative often. The most of the people in Khoy and neighboring area use aromatic water of Rosa damascena as "Golab" to treat stomachache, as sedative burns treatment and Natural Brightening Cleanser. In addition, it is used as flavor. For example, in 'Doogh' and 'Fereni' as traditional drink and food respectively and the essential oil of Rosa damascena is used in Perfumery. Golab has an important role especially in funeral of Azerian people. They use it for Wash graves and cooking "Halva" which is a traditional pastry for mourning ceremony. Different approaches used for medicinal preparations of plants are presented in Table 2 . The consumption of plants in Infusion form is the most frequently used way (40 Species), followed by Decoction (39 Species), Raw (20 Species), and Powder (8 Species) (Fig. 4).

Sadat-Hosseini et al. ${ }^{11}$ have carried out ethno-botanical studies in the south of Kerman Province. They have collected data from the native people. Majority of the are used as decoction followed by liniment, and infusion. As in our findings, most of the herbal preparations are consumed orally, whereas in a few cases Top mode is used. The oral mode of application is the most preferred form of herbal preparations among different ethical groups of Iran. However, according to Vijayakumar et al. most plant preparations are used in the form of paste (32\%), followed by powder (22\%), decoction and juice (20\%). Similarly, oral use too is the most frequently applied mode, followed by topical use. A perusal of the studies published reveals big differences between various cultures, in terms of mode of herbal preparations and application ways, but most are applied orally.

Data analysis (ICF and UV calculations)

The use of medicinal plants as conventional and modern drugs shows that they are acceptable. There may be some plants which are currently not used for medicinal purposes but may actually have medicinal effects (Kaya, 2006). In order to classify major health problems of interviewees, ailments are categorized Loading [MathJax]/jax/output/CommonHTML/jax.js were grouped into 11 categories based on the information gathered from the interviewee. Native people of Khoy 
use plants for medical purposes mostly for treatment of Gastro-intestinal diseases (e.g. gastric pains, stomach disorders, as carminative, constipation, diarrhea, hemorrhoids and laxatives and cases), cold, flu and fever (60 citations), Diabetes (32 citations), Skin diseases (28 citations), Nervous system, sedative (20 citations), Cardiovascular disease (16 citations), Diuretic (14 citations), Infections (13 citations), Rheumatic pain (12 citations), Respiratory/throat diseases and Cancer (6 citations) (Fig. 5).

Helichrysum arenarium (L.), Lepidium draba L., Mentha longifolia (L.) Huds., Thymus kotschyanus Boiss., Alcea kurdica, etc were reported to be among the plant remedies concerned for treating cold, flu and fever which had the highest ICF score (0.61). Diabetes was recorded to have the second highest ICF value (0.52), followed by Infections with a little interval recorded by its all images like the third group (ICF was 0.51 ). Respiratory and throat diseases were ranked as the fourth ailment with ICF value of 0.49 . the fifth uses of ICF values $(0.45)$ was recorded for skin diseases. The last citations of ranking of this research were reported for plants used to treat cardiovascular disease, Nervous system problems, Gastro-intestinal diseases, Diuretic and rheumatic pain with ICF value of $0.40,0.36,0.32,0.31,0.27$ respectively. Cancer had the least ICF values $(0.23)$. There is not any reliable similar study of this region with calculated ICF but this one (Table 3).

Table 3

Different ICPC-2 based categories of diseases, their related ICF values and popular plants in the related categories

\begin{tabular}{|lllll|}
\hline No. & Use category & Use Report & All use citations (\%) & ICF \\
\hline 1 & Cold, flu, fever & 60 & 19.87 & 0.61 \\
\hline 2 & Diabetes & 32 & 9.44 & 0.52 \\
\hline 3 & Infections & 13 & 4.3 & 0.51 \\
\hline 4 & Respiratory and throat diseases & 6 & 1.99 & 0.49 \\
\hline 5 & Skin diseases & 28 & 9.27 & 0.45 \\
\hline 6 & Cardiovascular disease & 16 & 5.3 & 0.40 \\
\hline 7 & Nervous system, sedative & 20 & 6.62 & 0.36 \\
\hline 8 & Gastro-intestinal diseases & 95 & 31.46 & 0.32 \\
\hline 9 & Diuretic & 14 & 4.64 & 0.31 \\
\hline 10 & Rheumatic pain & 12 & 3.97 & 0.27 \\
\hline 11 & Cancer & 6 & 1.99 & 0.23 \\
\hline
\end{tabular}

ICF values, obtained for the reported categories, demonstrate the degree of knowledge that is shared regarding the use of medicinal herbs for treatment of the ailment (AI Quran, 2009). The highest value of ICF is an important parameter in an ethnobotanical study and there are several studies. In the present study, it was found that the ICF values range between 0.61 and 0.23 . In some studies, ICF value was similar to our research for different ailments. F or example Hayta et al (2014) grouped ailments in 10 categories. they observed the highest ICF value for skin diseases (0.60) followed by respiratory and throat diseases (0.52). In another study in eastern Anatolia Mukemre et al. (2015) examined the diseases in 10 categories. In these categories, the highest ICF value was reported to be 0.62 while the lowest ICF value was reported to be 0.21 . but in the studies conducted in various areas of the Iberian Peninsula, 0.85 and 0.91 for a Portuguese and a Catalan region were the highest ICF value, respectively (Bonet and Valles, 2003; Camejo-Rodrigues et al., 2003). The gastrointestinal problems are the first use category in studies of different regions (Miraldi et al., 2001; Ghorbani, 2005; Ghorbani et al., 2011).

The most used categories of plants to treat an ailment, presented in this study, are the ones to treat Gastro-intestinal diseases (31.46\%, 64 species) and Cold (19.87, 24 species).

Use value is an important tool to select valuable medicinal plants of any region which further may be used for detailed pharmacological evaluation s for preparation of plant based medicines. Use values for each medicinal plant species was calculated in this research and described in Table 2. Thymus kotschyanus Boiss. exhibited maximum use value (0.58) among wild plants reported by informants followed by the Mentha longifolia (L.) Huds. (0.54), Peganum harmala L. (0.41), Matricaria chamomilla L. (0.41), Capparis spinosa L. (0.36) and Nasturtium officinale W. T. Aiton. (0.36). These plants are used at large scale in the study area. Among reported cultivated plants, Ocimum basilicum L had maximum use value (0.44) followed by Juglans regia L. (0.43), Mentha spicata L. subsp. Spicata (0.42), Rosa damascena (0.41) and Allium sativum L. (0.37). (Fig. 6). Having information of use value of a plants might be useful for determining the use reliability and pharmacological features of the related plants (Çakılcıoglu and Turkoglu, 2010). The current use of medicinal plants in conventional and modern drugs shows that they are actively used (Kaya, 2006).

Medicinal plants, reported in this study, were used for treatment diseases including cold, flu, fever, bronchitis, diabetes, eczema, respiratory, asthma, cardiac disorders, infections, liver disorders, constipation, diarrhea, rheumatism, bone problems, blood ailments like hypertension, high cholesterol, etc. Medicinal plants were also used as sedative, narcotic, intoxicant, antispasmodic, carminative, antiseptic, tonic, laxative, and diuretic agents. According to the results of this study, Azerian people of this region tend to use indigenous medicinal herbs.

Kahlik otu is the Azerian name of different species of Thymus (family Lamiaceae) and is frequently reported from different parts of Iran, especially in Khoy. Rosa damascena is a shrub that is cultivated especially in margins of gardens and is Widely used for wound care, Sedative, stomachache and constipation by native people. In addition, native people believe that the use of Gundelia tournefortii can decrease cholesterol, which is approved by Hajiadeh et al (2016).

Loading [MathJax]/jax/output/CommonHTML/jax.js

Page 10/14 
They expressed that Gundelia tournefortii could be an appropriate adjunctive medicinal plant to help reducing the major risk factors of CAD like cholesterol, LDL-C and BMI.

In addition, local people of Khoy use an expensive truffle, named "Donbalan" widely to use as antiinflammation, antioxidant and tonic. They believe that truffles, found in this region, are important source of protein and they use it for their health. White truffles, Terfezia boudieri Chatin. are found in Khoy and neighbouring area. It is one of seasonal temporary jobs to collect this kind of truffles for farmers which earn a proper income in some years. It's used to treat gastric cancer, hepatitis A, B, C, arthritis, bronchitis, asthma, stomach ulcers, blood pressure and cholesterol.

local Plant names

In different languages, local plant names differ because of local dialects. Because of vicinity of study area with Turkey, there could be similarities in plant local names. For example, the local names of Allium cepa (Sogan) in Izmir (Ugulu et al., 2009), Allium sativum (Sarimsak), Elaeagnus angustifolia (Ide), Rhus coriaria (Sumak) in Alasehir (Sargin et al., 2013), Allium cepa (Sogan), Allium sativum (Sarimsak), Gundelia turnefortii (KKangar), Ocimum basilicum (Reyhan) and Hordeum vulgare (Arpa) and in Espiy of Turkey (Polat et al, 2015) are the same with local names used in this region.

Ethnobotany and Ethnopharmacology are new emerging fields of research in Iran which are getting more attention from scientific community (Ghorbani et al., 2006). West Azerbaijan has rich flora, so medicinal plants are very important subjects in health care. Since a great number of medicinal plants are used by the population of this region, it seems that using these plant are somehow general preferences. It is very important to evaluate medicinal plants used in Khoy for following reasons: (1) Probable toxicity of some plants. Toxic plants or plant parts are not always recognized by market sellers. For instance, Datura stramoniumcould be toxic. (2) Besides, it's important to use effective dose of medicinal plants and Correct utilization methods of them.

\section{Conclusion}

In the research area, we found 82 plants referred to 31 plant families for medicinal use by informants of Khoy for the first time, which are used to treat many diseases. Asteraceae is the most used plant family and aerial parts are the most favored part for local users. Native people use these plants as decoctions or infusions after drying them. Therefore, they would use them during all seasons of the year. Most commonly used plants are Thymus kotschyanus Boiss., Mentha longifolia (L.), Ocimum basilicum L, Juglans regia L., Mentha spicata L. subsp. Spicata, Rosa damascene, Peganum harmala L., Matricaria chamomilla L., Allium sativum L., Capparis spinosa L. and Nasturtium officinale W. T. Aiton.

Plant parts used for the treatment of various illnesses include aerial parts, leaves, stems, roots, bark, milky latex, oil seeds, flowers, and fruits. Aerial parts were the most plant part (34), followed by fruit and seed (24), leaves (21), flower (9), latex (4) and root, rhizome, bulb (9) and a style respectively. These plants are used for the treatment of gastro-intestinal diseases, cardiovascular problems, diabetes, and skin diseases, cold and respiratory tract problem etc.

Relative importance value of plant species and informant consensus factor (ICF) for plants were calculated. The ICF values were found between 0.61 and 0.23 . Diseases with high ICF values according to reported disorders are cold, diabetes and gastrointestinal diseases. We perceived that these plants are used in different parts of the world for the treatment of the same or similar diseases. Some plants that mentioned in this paper, may be edible or have another application.

\section{References}

1. Abbasi AM, Hkan MA, Ahmad M, Zafar M. Ethnobotanical Aspects. Med. Plant Biodiverse. Lesser Himal Pak. 2012;13:220.

2. Azizi H, Keshavarzi M. 2015. Ethnobotanical study of medicinal plants of Sardasht, Western Azerbaijan, Iran, J Herb Drugs (An in to J Med Herbs), 6: 113119.

3. Bagcı Y. Ethnobotanical features of Aladaglar (Yahyalı Kayseri) and its vicinity. Herb J Syst Bot. 2000;7:89-94.

4. Bahmani M, Zargaran A, Rafieian-Kopaei M. Identification of medicinal plants of Urmia for treatment of gastrointestinal disorders. Rev Bras Farmacogn. 2014;24:468-80.

5. Brandao MGL, Pignal M, Romaniuc S, Grael CFF, Fagg CW. Useful Brazilian plants listed in the field books of the French naturalist Augusta de Saint-Hilaire (1779-1853(. J Ethnopharmacol. 2012;143:488-500.

6. Davis PH. Flora of Turkey and the East Aegean Islands. Edinburgh: 1965-1985, Edinburgh University Press; 1985. vol. 1-9.

7. Davis PH, Mill RR, Tan K. Flora of Turkey and the East Aegean Islands Vol 10, 1988. Edinburgh: Edinburgh University Press; 1988.

8. Dolatkhahi M, Yousofi M, Baghernejad J, Dolatkhahi A. Introductory study of the medicinal plants species of Kazeroon in Fars Province. J Herbal Drugs. 2010;3:47-56. (in Persian).

9. Ghorbani A, Langenberger G, Feng L, Sauerborn J. Ethnobotanical study of medicinal plants utilized by Hani ethnicity in Naban river Watershed National Nature Reserve, Yunnan, China. J Ethnopharmacol. 2011;134:651-67.

10. Ghorbani A, Naghibi F, Mosaddegh M. Ethnobotany, Ethnopharmacology and drug discovery. Iran J Pharm Sci. 2006;2:109-18.

11. Hajizadeh-Sharafabad F, Alizadeh M, Mohammadzadeh S, Alizadeh-Salteh H, Kheirourie S, S. Effect of Gundelia tournefortii L. extract on lipid profile and TAC in patients with coronary artery disease: A double-blind randomized placebo controlled clinical trial. J Herb Med. 2016;6(2):59-66.

12. Hayta S, Polat R, Selvi S. Traditional uses of medicinal plants in Elazığ (Turkey (. J Ethnopharmacol. 2014;154:613-23.

13. Hoft M, Barik SK, Lykke AM. 1999. Quantitative Ethnobotany. Applications of Multivariate and Statistical Analyses in Ethnobotany, People and Plants Working Paper 6. Unesco, Paris. p 3.

Loading [MathJax]/jax/output/CommonHTML/jax.js 
14. Iranmanesh M, Najafi Sh, Yousofi M. Ethnobotany survey of medicinal plant of Sistan region. J Herbal Drugs. 2010;2:61-8. (in Persian).

15. Kaya G. Discussion of P\&P model used for estimating option value of forest ecosystems as medicinal plant resources. J Barton Fac Forest. 2006;8:2332.

16. Miraldi E, Ferri S, Mostaghimi V. Botanical drugs and preparations in the traditional medicine of West Azerbaijan (Iran (. J Ethnopharmacol. 2001;75:7787.

17. Mosaddegh M, Naghibi F, Moazzeni H, Pirani A, Esmaeili S. Ethnobotanical survey of herbal remedies traditionally used in Kohghiluyeh va Boyer Ahmad province of Iran. J Ethnopharmacol. 2012;141:80-95.

18. Mood SG. Contribution to some ethnobotanical aspects of Birjand flora (Iran (. Pak J Bot. 2008;40:1783-91.

19. Mükemre M, Behçet L, Çakılcıoğlu U. Ethnobotanical study on medicinal plants in villages of Çatak (Van-Turkey(. J Ethnopharmacol. 2015;166:361-74.

20. Naghibi F, Mosaddegh M, Mohammadi., Motamed S, Ghorbani A. Labiatae family in folk medicine in Iran: from ethnobotany to pharmacology. Iran J Pharm Res. 2005;2:63-79.

21. Özdemir E, Alpınar K. An ethnobotanical survey of medicinal plants in western part of central Taurus Mountains: Aladaglar (Nigde-Turkey). J Ethnopharmacol. 2015;166:53-65.

22. Phillips O, Gentry AH. The useful plants of Tambopata, Peru: I. Statistical hypothesis tests with a new quantitative technique. Econ Bot. 1993;47:15-32.

23. Polat R, Çakılcıoğlu U, Kaltalığlu K, Ulusan MD, Türkmen Z. An ethnobotanical study on medicinal plants in Espiye and its surrounding (Giresun-Turkey). J Ethnopharmacol. 2015;163:1-11.

24. Rechinger, editor, KH, 1965-2008., Flora Iranica, No. 1-164, Akademische Druch-u, Verlagsanstat, Graz-Austria.

25. Sadeghi Z, Kuhestani K, Abdollahi V, Mahmood A. Ethno pharmacological studies of indigenous medicinal plants of Saravan region, Baluchistan, Iran. J Ethnopharmacol. 2014;153:111-8.

26. Sadeghi Z, Akaberi M, Sobhkhizi A, Sahebkar A, Emami SA. Evaluation the ethno-pharmacological studies in Iran during 2004-2016: A systematic review. J Cell Physiol. 2018;233:914-23.

27. Sadat-Hosseini M, Farajpour M, Boroomand N, Solaimani-Sardou F. 2017. Ethno pharmacological studies of indigenous medicinal plants in the south of Kerman, Iran, J Ethnopharmacol, 199: 194-204.\&\#8206.

28. Sargın SA, Akçicek E, Selvi S. An ethnobotanical study of medicinal plants used by the local people of Alaşehir (Manisa) in Turkey. J Ethnopharmacol. 2013;150:860-74.

29. Sharififar F, Koohpayeh A, Motaghi MM, Amirkhosravi A, Nasab P, et al. Study the ethnobotany of medicinal plants in Sirjan, Kerman Province, Iran. J Herbal Drugs. 2010;3:19-28.

30. Trotter RT, Logan MH. Informant consensus: a new approach for identifying potentially effective medicinal plants. In: Etkin NL, editor. Plants in Indigenous Medicine and Diet, Behavioural Approaches. Bredford Hills: Redgrave Publishing Company; 1986.

31. Ugulu I, Baslar S, Yorek N, Dogan Y. The investigation and quantitative ethnobotanical evaluation of medicinal plants used around Izmir province, Turkey. J Med Plants Res. 2009;3:345-67.

32. Yousofi M. Flora of Iran. Tehran: Payam Noor University Publication; 2007. p. 93. (in Persian).

\section{Figures}




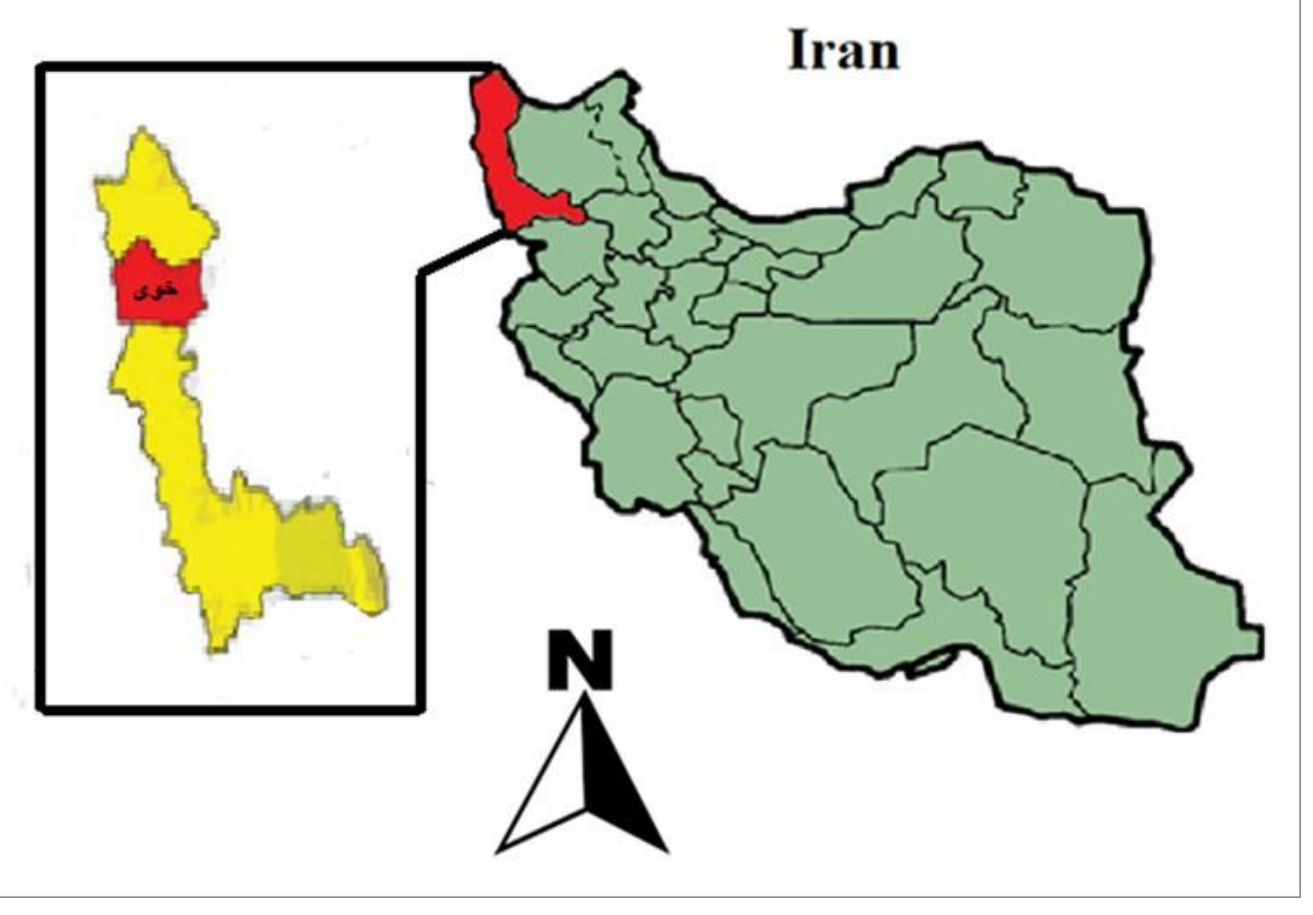

Figure 1

Study area map showing Khoy city, West Azerbaijan Province, Iran.

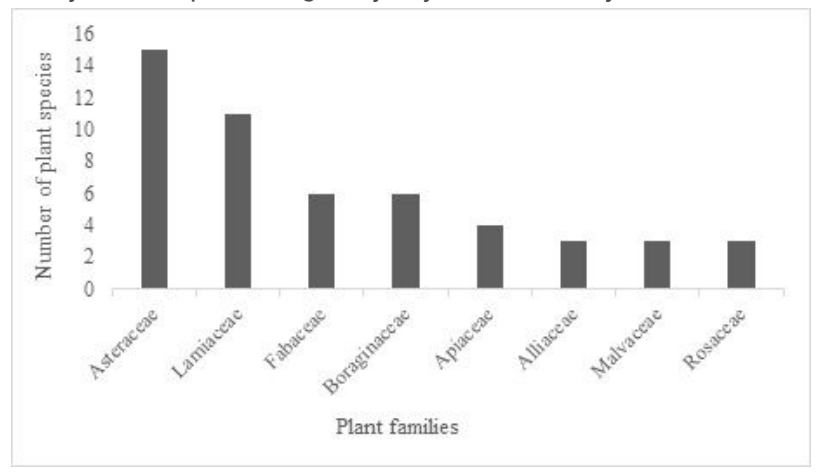

Figure 2

Number of cited plants from respective plant family

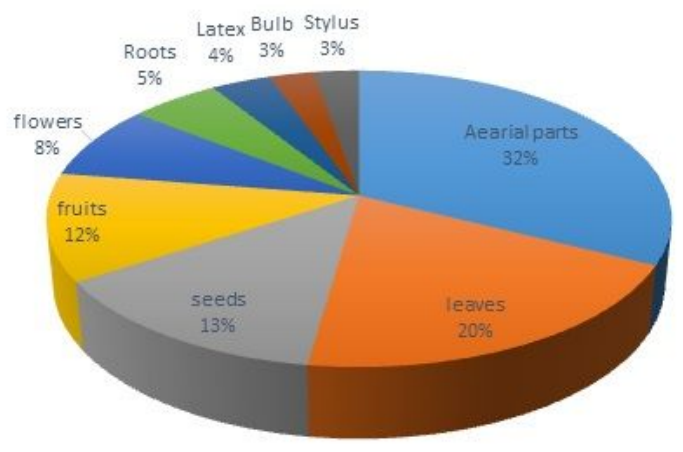

Figure 3

Different plant parts used by people and their related percentages in the study area. 


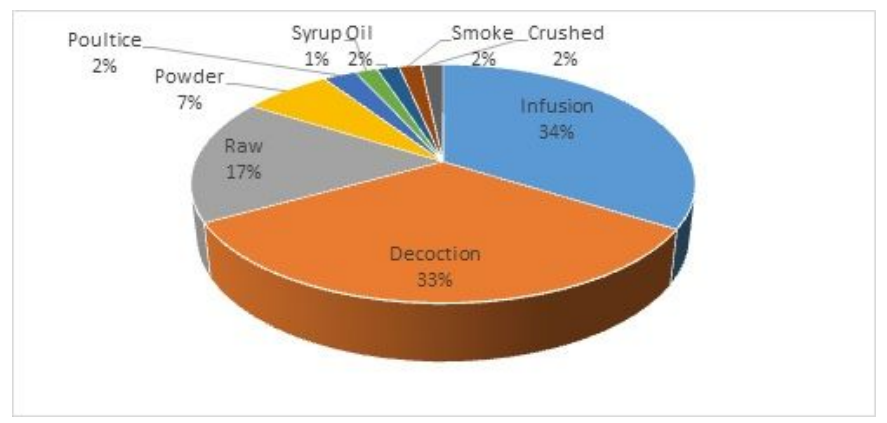

Figure 4

Mode of preparations and their percentages.

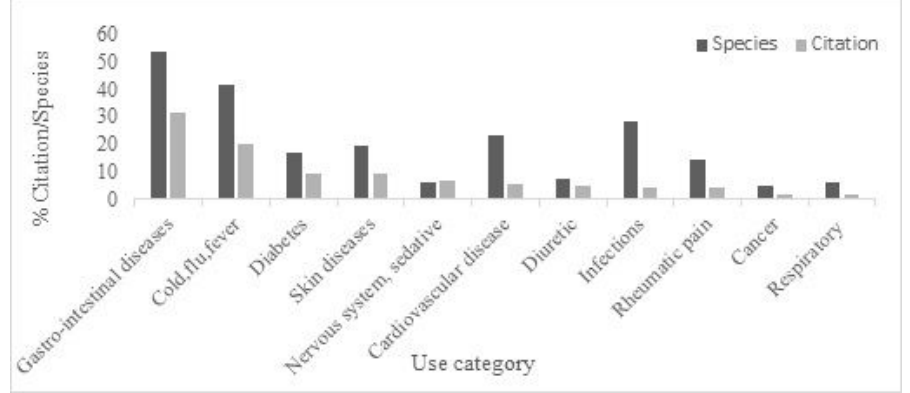

\section{Figure 5}

Percentage of species and citation in each use category.

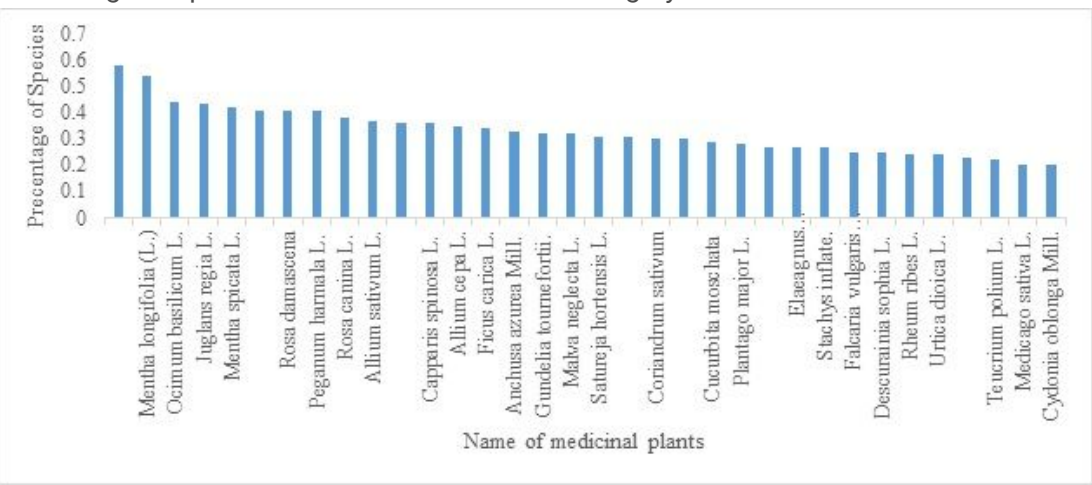

Figure 6

Medicinal plants with the most use 